\title{
Some Fermi Surface Properties of Double-Exchange Interaction Systems
}

\author{
C. D. $\mathrm{Hu}$ \\ Department of Physics and NCTS at TPE, National Taiwan University, Taipei, Taiwan, Republic of China
} (Received 1 March 2004; published 5 October 2004)

\begin{abstract}
We study the photoemission spectrum of the double-exchange (DE) interaction systems. The DE Hamiltonian can be transformed into a simple form consisting of fermions and Schwinger bosons. We apply the gauge-field model and calculate the Green's function of the gauge field, fermions, and bosons. The imaginary part of the Green's function of an electron has an asymmetrical peak with strong temperature dependence. This can explain why the shape of the angle-resolved photoemission spectra of manganites near the Fermi surface is very different from that of Fermi liquid. We also show why the position of the Fermi surface is not sensitive to temperature.
\end{abstract}

The fundamental mechanism in perovskite manganites $\left(\mathrm{La}_{1-x} A_{x} \mathrm{MnO}_{3}\right.$, where $A$ is the divalent element and $x$ is the doping concentration) is the double-exchange (DE) interaction. It [1-3] is responsible for the intimate correlation between transport and magnetism. More recent studies [4] revealed a complex phase diagram and very rich physics. Though most physical phenomena are yet to be explained quantitatively, it seems that the properties of the manganites near optimum doping at low temperature where DE is clearly the dominant mechanism is well understood. Intuitively, one can conceive the following picture. The system is in the ferromagnetic state with spins coupled to each other via conduction electrons. The conduction electrons with spins aligned form Fermi liquid. However, recent angle-resolved photoemission spectrum (ARPES) experiments on several manganite system experiments [5-7] showed some very interesting results that cannot be explained by the above simple picture. Among them, two stood out. The first one is the spectrum near the Fermi surface. It has a strong temperature dependence and a shape completely different from that of a Fermi liquid, e.g., gold. The second important discovery is the position of the Fermi surface. At very low temperature all the spins of the conduction electrons are aligned. Hence, there is only one big Fermi sphere. Near $T_{C}$, there should be two smaller Fermi spheres. However, the experiment by Saitoh and co-workers [7] showed only a $4 \%$ shift in the position of the Fermi surface, instead of the 30\% [8] expected by conventional Fermi liquid theory.

The goal of this Letter is to study DE in detail. The ARPES results set an important criterion of validity for our approach. Orbital ordering, Yahn-Teller effect, polaronic effect, and inhomogeneities such as mesoscopic textures [9], though very interesting, make analysis difficult. We intentionally focus our attention on the region where DE is the dominant factor, namely, near optimum doping and away from $T_{C}$.

In the past ten years, there has been significant progress in the study of DE, notably the applying the Schwinger boson model [10-15]. However, most works have used Holstein-Primakoff (HP) transformation in later calculations. This approximation is good only for large spins and low temperature. Another approach $(1 / S$ expansion), proposed by Shannon [13], is promising. Here, we try to attack the problem from still another direction and hopefully develop a method applicable to a wide range of conditions. The major difficulty of the calculation involving the Schwinger bosons is the constraints. From the study of high $T_{C}$ materials, we find that the gauge-field model $[16,17]$ is suitable for treating fields with constraints. Additionally, ARPES has been widely used to study the existence or the properties of quasiparticles in strongly correlated systems [18]. The analysis on manganites will be helpful to the study of other many-body systems. Finally, according to our study and experimental data [7], the 3D system is very different from the 2D system. So our results probably cannot be compared with those experimental data [7] or calculations [19] for 2D systems.

We started with the double-exchange Hamiltonian:

$$
H=-t \sum_{\mathbf{k}, \sigma} c_{\mathbf{i} \sigma}^{\dagger} c_{\mathbf{j} \sigma}+J \sum_{\mathbf{i}} \mathbf{S}_{\mathbf{i}} \bullet \sigma_{\mathbf{i} \cdot \chi}
$$

The first term describes the hopping of the holes between $\mathrm{Mn}^{+3}$ and $\mathrm{Mn}^{+4}$ sites. The second term, where $\mathbf{S}_{i}$ denotes the manganite ion spins and $\sigma_{i}$ the hole spins, is the Hund's coupling. The level spacing $J$, of the order of $\mathrm{eV}$, is the largest energy in the system and hence cannot be treated perturbatively. For this reason, Kubo and Ohata [8] used projection operators to apply a constraint to holes. Hoppings can occur only at those sites where the local spins are antiparallel to those of the holes. Thus, the Hund's coupling can be removed from the Hamiltonian because all of the states have the same coupling energy. Further progress is made by applying the slave-fermion method to holes to separate the spins from the charges: $c_{i \downarrow(\uparrow)}=f_{i} \alpha_{i \downarrow(\uparrow)}$, where $f_{i}$ is the fermion operator and $\alpha_{i \uparrow(\downarrow)}$ the spin-up (spin-down) spinon operator. Then, 
Schwinger bosons are introduced to represent the total spins: $\quad b_{\uparrow}^{\dagger}|S-1 / 2, m-1 / 2\rangle=\sqrt{(2 S+1)(S+m)}|S, m\rangle$ and $\quad b_{\downarrow}^{\dagger}|S-1 / 2, m+1 / 2\rangle=\sqrt{(2 S+1)(S-m)}|S, m\rangle$.

$$
G_{i j}^{a}\left(i \omega_{n}, \mathbf{q}\right)=\left(\frac{1}{\Pi^{f}\left(i \omega_{n}, \mathbf{q}\right)+\Pi^{\uparrow}\left(i \omega_{n}, \mathbf{q}\right)+\Pi^{\downarrow}\left(i \omega_{n}, \mathbf{q}\right)}\right)_{i j}
$$

The Hamiltonian now becomes

$$
H=-\frac{t}{2 S+1} \sum_{\mathbf{i}, \mathbf{j}} f_{\mathbf{i}}^{\dagger}\left(b_{\mathbf{j} \uparrow}^{\dagger} b_{\mathbf{i} \uparrow}+b_{\mathbf{j} \downarrow}^{\dagger} b_{\mathbf{i} \downarrow}\right) f_{\mathbf{j}},
$$

with the constraint on each site for slave fermions and Schwinger bosons:

$$
b_{\mathbf{i} \uparrow}^{\dagger} b_{\mathbf{i} \uparrow}+b_{\mathbf{i} \downarrow}^{\dagger} b_{\mathbf{i} \downarrow}+f_{i}^{\dagger} f_{i}=2 S .
$$

Now the gauge-field model was introduced to decouple the fermions and bosons [19]. The constraints can be taken into account with the Lagrange undetermined multipliers, which, in turn, can be absorbed into the temporal part of the gauge field. If the phase fluctuation of the gauge field is considered, then the Lagrangian has the following form in the continuum limit [20]:

$$
\begin{aligned}
\mathcal{L}= & \sum_{\sigma=\uparrow, \downarrow} \int d^{3} \mathbf{r}\left[f^{\dagger}\left(\frac{\partial}{\partial \tau}-\mu_{f}+i a_{0}\right) f\right. \\
& +\frac{1}{2 m_{f}} f^{\dagger}\left(\frac{\nabla}{i}-\mathbf{a}\right)^{2} f+b_{\sigma}^{\dagger}\left(\frac{\partial}{\partial \tau}-\mu_{b}+i a_{0}\right) b_{\sigma} \\
& \left.+\frac{1}{2 m_{\sigma}} b_{\sigma}^{\dagger}\left(\frac{\nabla}{i}-\mathbf{a}\right)^{2} b_{\sigma}\right],
\end{aligned}
$$

where $\hbar$ had been set to unity, $\mu_{f}$ and $\mu_{b}$ are the chemical potentials of fermions and bosons, respectively, and the effective masses of fermions and bosons were determined by the mean-field approximation of Eq. (2): $m_{f}=(2 S+$ 1) $/ 2 S a^{2} t$ and $m_{\uparrow}=m_{\downarrow}=m_{b}=(2 S+1) / a^{2} x t$, with $a$ being the lattice constant and $x$ the hole concentration. The mass of bosons is an order magnitude greater than that of fermions. In order to understand the system, one would like to calculate the Green's function of electrons, or, in our case, the holes. It is a convolution of the Green's functions of fermions and spinons. For the relations between the spinons and Schwinger bosons, we found that in the restricted vector space $|S, m\rangle$ and $\left|S-1 / 2, m^{\prime}\right\rangle$, where $S=2$, the spinons have functions: $\alpha_{\downarrow} \mid S-1 / 2, m-$ $1 / 2\rangle=\sqrt{(S+m)}|S, m\rangle \quad$ and $\quad \alpha_{\uparrow}|S-1 / 2, m+1 / 2\rangle=$ $\sqrt{(S-m)}|S, m\rangle$, which are the same as those of Schwinger bosons. Hence, the Green function of holes can written as a convolution of that of fermions and Schwinger bosons:

$$
G_{\downarrow}^{h}\left(i \omega_{n}, \mathbf{k}\right)=\frac{1}{\beta} \sum_{m, \mathbf{q}} G^{f}\left(i \omega_{n}+i \omega_{m}, \mathbf{k}+\mathbf{q}\right) G^{\dagger}\left(i \omega_{m}, \mathbf{q}\right),
$$

where $G^{f}\left(i \omega_{n}, \mathbf{k}\right)$ and $G^{\uparrow}\left(i \omega_{m}, \mathbf{q}\right)$ are the fermion and spin-up Schwinger boson Green functions. Both of them are related to the Green function of the gauge field, $-\left\langle\tilde{T} a_{i, \mathbf{q}}(\tau) a_{j, \mathbf{q}}^{\dagger}\right\rangle[17]$. where the superscript of the current-current correlation function $\Pi^{\gamma}\left(i \omega_{n}, \mathbf{q}\right)(\gamma=f, \uparrow, \downarrow)$ denotes the fermions and the spin-up and spin-down Schwinger bosons, and the subscripts $i$ and $j$ denote the spatial components.

Since $\Pi \gamma\left(i \omega_{n}, \mathbf{q}\right)$ 's are related to all the important physics, a little elaboration is in order. The temporal component $\left\langle\tilde{T} a_{0, \mathbf{q}}(\tau) a_{0, \mathbf{q}}^{\dagger}\right\rangle$ is a density-density correlation function. Its effect is limited by screening and hence can be neglected. For the spatial components, we have

$$
\begin{aligned}
\Pi_{i j}^{\gamma}\left(i \omega_{n}, \mathbf{q}\right)= & \frac{n_{\gamma}}{m_{\gamma}} \delta_{i j}+\frac{1}{N} \sum_{\mathbf{p}} \frac{(2 p+q)_{i}(2 p+q)_{j}}{4 m_{\gamma}^{2}} \\
& \times \frac{n_{\gamma}(\mathbf{p})-n_{\gamma}(\mathbf{p}+\mathbf{g})}{i \omega_{n}-\varepsilon_{\gamma, \mathbf{p}+\mathbf{q}}+\varepsilon_{\gamma, \mathbf{p}}},
\end{aligned}
$$

where $\varepsilon_{\gamma, \mathbf{p}}=p^{2} / 2 m_{\gamma}$ is the energy of the fermions, spinup and spin-down Schwinger bosons, $n_{\gamma}$, and $n_{\gamma}(\mathbf{p})$ are the average number per site and distribution functions, respectively. As $i \omega_{n}=0$ and $\mathbf{q} \rightarrow 0$, the two terms on the right-hand side (rhs) of Eq. (7) cancel each other for both the longitudinal part and the transverse part of $\Pi_{i j}^{f}\left(i \omega_{n}, \mathbf{q}\right)$. The situation of bosons is different if there is long-range order. There is cancellation for the longitudinal part but not for the transverse part. To illustrate this point, consider the ferromagnetic phase at $T=0 \mathrm{~K}$ with all the ion spins pointing upward. There is "condensation" $[21,22]$ at zero-momentum state: $n_{\uparrow}(0) \neq 0$ due to the abundance of spin-up bosons, $\left\langle b_{\uparrow, i}^{\dagger} b_{\uparrow, i}\right\rangle=2 S-x$. The second term on the rhs of Eq. (7) contributes a term $n_{\uparrow}(0) \delta_{i j} / m_{b}$ to the longitudinal $\Pi_{i j}^{\uparrow}\left(i \omega_{n}, \mathbf{q}\right)$ but nothing to the transverse one. This important difference results in the cancellation in the denominator of the longitudinal part of Eq. (6). Therefore, we can neglect the transverse part. From now on, we concerned ourselves only with the longitudinal part of the current-current correlation function, which is to be denoted as $\Pi^{\gamma}(\omega, \mathbf{q})$.

It is helpful to understand the physics by studying the expressions of the imaginary part of $\Pi^{\gamma}(\omega, \mathbf{q})$ :

$$
\begin{aligned}
\operatorname{Im} \prod^{f}(\omega+i \delta, \mathbf{q})= & -\frac{a^{3}\left(m_{f} \omega / q\right)^{2}}{8 \pi m_{f} q} \sum_{\sigma= \pm} \sigma \theta\left(k_{F}^{2}-k_{f \sigma}^{2}\right) \\
& \times\left(k_{F}^{2}-k_{f \sigma}^{2}\right),
\end{aligned}
$$




$$
\begin{aligned}
\operatorname{Im} \Pi^{\dagger(\downarrow)}(\omega+i \delta, \mathbf{q})= & \frac{\pi q^{2} n_{\uparrow(\downarrow)}(0)}{4 m_{b}^{2}}\left[\delta\left(\omega-\varepsilon_{b}(\mathbf{q})\right)\right. \\
& \left.-\delta\left(\omega+\varepsilon_{b}(\mathbf{q})\right)\right]-\frac{a^{3}\left(m_{b} \omega / q\right)^{2}}{8 \pi m_{b} q \lambda_{T}^{2}} \\
& \times \ln \frac{1-\exp \left(-\beta k_{b-}^{2} / 2 m_{b}\right)}{1-\exp \left(-\beta k_{b+}^{2} / 2 m_{b}\right)},
\end{aligned}
$$

where $\theta(x)$ is the step function, $k_{f(b) \pm}=m_{f(b)} \omega / q \pm q / 2$, and $\lambda_{T}=1 / \sqrt{2 m_{b} k_{B} T}$ is the thermal wavelength. Clearly, the fermion part comes from particle-hole excitation and the boson part from scattering. The characteristic Bose-Einstein distribution function is manifest in Eq. (8b). The first term is the contribution from the "condensate." The divergence of the second term is also due to $n_{b}(\mathbf{p})$. It would have been smoothed out if higher order terms were considered. Nevertheless, the divergence has little effect on our result because it appears in the denominator of $G^{a}(\omega, \mathbf{q})$. Other than divergence, the ratio of $\operatorname{Im} \Pi^{\dagger(\downarrow)}(\omega, \mathbf{q})$ to $\operatorname{Im} \Pi^{f}(\omega, \mathbf{q})$ is approximately $1 /\left(k_{F} \lambda_{T}\right)^{2}$, which is small in the temperature range we are interested in. As a result, the fermion part is much more important in the following calculation. However, one must remember that it is bosons which make the transverse component of $\Pi(\omega, \mathbf{q})$ negligible.

We analyzed the correlation functions and found that $\operatorname{Re} \Pi^{f(b)}(\omega, \mathbf{q})$ is of the order $x / m_{f} \simeq 2 S / m_{b}$ for finite $q$, and $\operatorname{Im} \Pi^{f}(\omega, \mathbf{q}) \sim\left(x / m_{f}\right)\left(m_{f} \omega / q\right)^{2} / k_{F} q$ for finite $q$ and $\omega$. For $q \ll k_{f}$ and $\omega=\xi k_{F} q / m_{f}$,

$$
\operatorname{Im} \Pi^{f}(\omega, \mathbf{q}) \sim x \xi^{3} / m_{f} .
$$

Now we are in a position to calculate the self-energies of fermions and bosons (and then the Green function of holes). They were denoted by $\Sigma^{f}\left(i \omega_{n}, \mathbf{q}\right)$ and $\Sigma^{b}\left(i \omega_{n}, \mathbf{q}\right)$, respectively. Take the former as an example,

$$
\begin{aligned}
\Sigma^{f}\left(i \omega_{n}, \mathbf{q}\right)= & -\frac{1}{N \beta} \sum_{m, \mathbf{p}} \frac{\left(2 p_{\|}+q\right)^{2}}{4 m_{f}^{2}} G_{0}^{f}\left(i \omega_{n}+i \omega_{m}, \mathbf{p}\right. \\
& +\mathbf{q}) G^{a}\left(i \omega_{m}, \mathbf{p}\right),
\end{aligned}
$$

where $p_{\|}$is the component parallel to $\mathbf{q}$ and $G_{0}^{f}\left(i \omega_{n}, \mathbf{p}\right)$ is the zeroth order Green function of fermions. Since the real part of the self-energy only shifts the particle energy, we concentrated on the imaginary part. The imaginary part of the Green function, $G_{0}^{f}(z, \mathbf{p})$, is a Dirac delta function; the frequency sum can be done easily:

$$
\begin{aligned}
\operatorname{Im} \Sigma^{f(b)}(\omega+i \delta, \mathbf{q})= & \sum_{\mathbf{p}} \frac{\left(2 p_{\|}+q\right)^{2}}{4 m_{f}^{2}}\left[n_{b}\left(\varepsilon_{f(b), \mathbf{p}+\mathbf{q}}-\omega\right)\right. \\
& \left. \pm n_{f(b)}\left(\varepsilon_{f(b), \mathbf{p}+\mathbf{q}}\right)\right] \operatorname{Im} G^{a}\left(\varepsilon_{f(b), \mathbf{p}+\mathbf{q}}\right. \\
& -\omega-i \delta, \mathbf{p}),
\end{aligned}
$$

where $+(-)$ is for fermions (bosons). We can make a quite accurate estimation of $\operatorname{Im} \Sigma^{f}(\omega, \mathbf{q})$ near the Fermi surface; i.e., $q \approx k_{F}$ and $|\omega| \leq k_{B} T$. It should be noted that the factor $\left[n_{b}\left(\varepsilon_{f, \mathbf{p}+\mathbf{q}}-\omega\right)+n_{f}\left(\varepsilon_{f, \mathbf{p}+\mathbf{q}}\right)\right]$ in Eq. (10) imposes a severe restriction on the summation of $\mathbf{p}$. $n_{b}\left(\varepsilon_{f, \mathbf{p}+\mathbf{q}}-\omega\right)$ is negative for nearly all the negative $\varepsilon_{f, \mathbf{p}+\mathbf{q}}$. Thus the factor gives vanishingly small values unless $\left|\varepsilon_{f, \mathbf{p}+\mathbf{q}}\right| \lesssim \omega, k_{B} T$. (The similar can also be said of bosons.) The restriction greatly reduced the magnitudes of $\operatorname{Im} \Sigma^{f}(\omega, \mathbf{q})$. Physically, it means that for lowlying excitations, only those fermions near the Fermi surface and those bosons in low-lying states make significant contributions to self-energy. Effectively, the domain of $\mathbf{p}$ is centered around $k_{F}$ because of the restriction $\left|\varepsilon_{f, \mathbf{p}+\mathbf{q}}\right| \lesssim \omega, k_{B} T$. Because of the properties of $\operatorname{Im} \Pi^{f}(\omega, \mathbf{q})$ in (9), we found that the main contribution in Eq. (10) came from the small $p$ region. Thus, near the Fermi surface, $\operatorname{Im} \Sigma^{f}(\omega, \mathbf{q}) \propto \omega^{2} / \varepsilon_{F}$, the same as the conventional Fermi liquid. For the bosons, with the help of relations (8)-(11), we found that $\operatorname{Im} \Sigma^{\dagger(\downarrow)}(\omega, \mathbf{q}) \sim$ $\frac{m_{f}}{m_{b}} \operatorname{Im} \Sigma f(\omega, \mathbf{q})[23]$.

The photoemission spectrum is proportional to $|M|^{2} \operatorname{Im} G_{R}^{e}(\Omega, \mathbf{k})$. Here $M$ is the coupling matrix of photons and electrons. We did not account for the matrix since it required electron wave functions from band calculation. However, we can study the form of the imaginary part of Green's function where the main feature of the spectrum is contained. Having performed the frequency summation in Eq. (5) and treated $\operatorname{Im} G^{\dagger}(z, \mathbf{q})$ as a Dirac delta function due to the smallness of $\operatorname{Im} \Sigma^{\dagger}(\omega, \mathbf{q})$, we obtain

$$
\begin{aligned}
\operatorname{Im} G_{\downarrow R}^{h}(\Omega, \mathbf{k})= & \pi n_{b}(0) \operatorname{Im} G_{R}^{f}(\Omega, \mathbf{k})+\pi \sum_{\mathbf{q}}\left[n_{b}\left(\varepsilon_{b, \mathbf{q}}\right)\right. \\
& \left.+n_{f}\left(\varepsilon_{b, \mathbf{q}}+\Omega\right)\right] \operatorname{Im} G_{R}^{f}\left(\varepsilon_{b, \mathbf{q}}\right. \\
& +\Omega, \mathbf{q}+\mathbf{k}),
\end{aligned}
$$

where $\Omega$ is the energy of holes measured from the Fermi surface and the first term on the rhs is the contribution from the condensate. At present, we do not have an applicable theory to calculate $n_{b}(0)$ because our Schwinger bosons are interacting strongly with the gauge field. However, this difficulty was circumvented in the following way. We noticed the total weight of $\operatorname{Im} G_{\downarrow}^{h}(\Omega, \mathbf{k})$ has to be equal to $\pi$. Hence, we determine $n_{b}(0)$ by requiring this condition be fulfilled.

Since we are interested in the spectrum near the Fermi surface, we set $|\mathbf{k}|=k_{F}$ and calculated $\operatorname{Im} G_{\downarrow R}^{h}(\Omega, \mathbf{k})$. The results are shown in Fig. 1 with $t=0.2 \mathrm{eV}$ for doping concentration $x=0.2$ and $x=0.3$, with the former being shifted toward the left by $1 \mathrm{eV}$. The dotted lines are those at $20 \mathrm{~K}$ and the solid lines at $80 \mathrm{~K}$. The peaks come mainly from the first term of Eq. (12) and the broad tails from the second. As expected, the ones at $20 \mathrm{~K}$ have sharper peaks. A more interesting feature is the asym- 


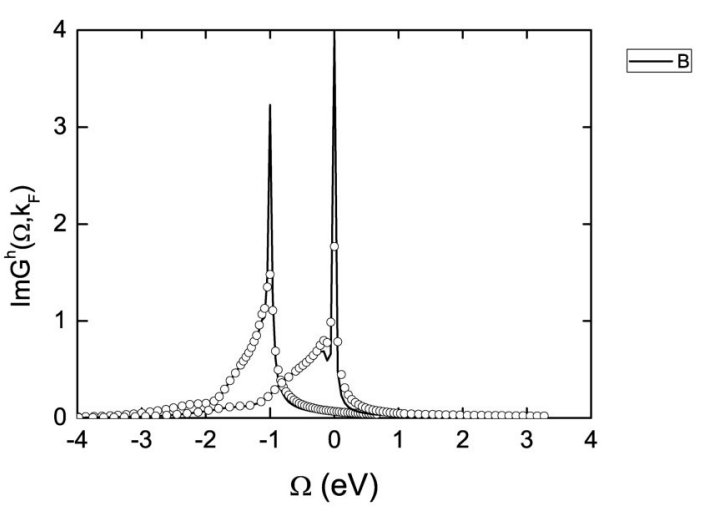

FIG. 1. $\operatorname{Im} G_{\mid R}^{h}(\Omega, \mathbf{k})$ versus $\Omega$ at doping concentration $x=$ 0.2 and $x=0.3$. The dotted lines are those at $20 \mathrm{~K}$ and the solid lines at $80 \mathrm{~K}$. Those of $x=0.2$ were shifted $1 \mathrm{eV}$ to the left for readability.

metry. The peaks have very broad tails for $\Omega<0$. (Since our approach has particle-hole symmetry, the properties of $\Omega<0$ holes can be applied to $\Omega>0$ electrons.) The asymmetry of $\operatorname{Im} G_{\downarrow}^{h}(\Omega, \mathbf{k})$ can be deduced in the following way. For $\Omega<0$, the factor $\left[n_{b}\left(\varepsilon_{b, \mathbf{q}}\right)+n_{f}\left(\varepsilon_{b, \mathbf{q}}+\Omega\right)\right]$ requires $\left|\varepsilon_{b, \mathbf{q}}\right| \lesssim k_{B} T$ or $\mathbf{q} \lesssim \sqrt{2 m_{b} k_{B} T}$. The restriction reduced the magnitude of $\operatorname{Im} G^{h}(\Omega, \mathbf{q})$. On the other hand, there is no such restriction on $\mathbf{q}$ when $\Omega>0$, and $\operatorname{Im} G_{\downarrow R}^{h}(\Omega, \mathbf{k})$ decreases as $\varepsilon_{f} / \Omega^{2}$ for large $\Omega$. For the conventional Fermi liquid, the spectrum has a jump at the Fermi surface even at room temperature. Here, as the photon energy increases, the intensity of photoemission increases smoothly due to the broad tail and saturates at the Fermi surface. It is also sensitive to temperature variation because the weight of the first term in Eq. (12) decreases with increasing temperature.

A very interesting difference between our results and those using HP transformation $[9,10]$ should be noted. While the broadening of Fermi edge given by HP transformation is of the order of the spin wave energy, ours is much larger. It comes from the interaction with the gauge field, which, in turn, comes from the interaction between fermions and bosons. However, at very low temperature, the first term of Eq. (12), or the condensation term, dominates. The broadening reduces to the order of $k_{B} T$ and agrees with the result of HP transformation. The difference strengthened our point of view that the gauge-field model is better than the HP transformation for manganites because the latter cannot give such broadening shown in ARPES. However, the relation between the mean-field approximation and the gauge-field model of Schwinger bosons is a topic worth studying.

Our calculation can also explain the result that the position of Fermi surface is insensitive to temperature. One can see easily that there is really only one Fermi sphere and that it is that of the slave fermion imbedded in a host of spins. In fact, this is a strong experimental evidence of spin-charge separation. Finally, we are able to provide a clear picture of DE systems. The spins of holes are coupled strongly to the ion spins and separated from their charges. Hence, they are not good quantum numbers. The unusual shape of the spectrum at the Fermi surface is due to the strong interaction between fermions and spins. This results in a temperature-sensitive Fermi edge and excitations no longer restricted to the Fermi surface.

The author is indebted to C.-Y. Mou for inspiring discussions. This work was support in part by NSC of Taiwan, Republic of China, under Contract No. NSC 912112-M-002-038.

[1] C. Zener, Phys. Rev. 82, 403 (1951).

[2] P.W. Anderson and H. Hasegawa, Phys. Rev. 100, 675 (1955).

[3] G. H. Jonker and J. H. Santen, Physica (Amsterdam) 16, 337 (1950).

[4] R. M. Kusters et al., Physica (Amsterdam) 155B, 362 (1989); S. Jin et al., Science 264, 413 (1994); Y. Tokura et al., J. Phys. Soc. Jpn. 63, 3931 (1994).

[5] D. D. Sarma et al., Phys. Rev. B 53, 6873 (1996).

[6] D. S. Dessau et al., Phys. Rev. Lett. 81, 192 (1998).

[7] T. Saitoh et al., Phys. Rev. B 62, 1039 (2000).

[8] K. Kubo and N. Ohata, J. Phys. Soc. Jpn. 33, 21 (1972); 33, 929 (1972).

[9] Andrea Damascelli et al., Rev. Mod. Phys. 75, 473 (2003); M. B. Salamon and M. Jaime, Rev. Mod. Phys. 73, 583 (2001).

[10] Sanjoy K. Sarkar, J. Phys. Condens. Matter 8, L515 (1996).

[11] G. Khaliullin and R. Kilian, Phys. Rev. B 61, 3494 (2000); R. Kilian and G. Khaliullin, ibid. 58, 11841 (1998).

[12] A. Weisse et al., Phys. Rev. B 64, 054406 (2001).

[13] N. Shannon, Phys. Status Solidi (b) 236, 494 (2003). However, his interaction term is still of the order $S^{0}$.

[14] S.-Q. Shen et al., Phys. Rev. B 62, 5829 (2000).

[15] S. Ishizaka and S. Ishihara, Phys. Rev. B 59, 8375 (1999).

[16] G. Baskaran and P.W. Anderson, Phys. Rev. B 37, 580 (1988).

[17] L. B. Ioffe and A. I. Larkin, Phys. Rev. B 39, 8988 (1989).

[18] J.W. Allen, Solid State Commun. 123, 469 (2002).

[19] Adriana Moreo et al., Phys. Rev. Lett. 83, 2773 (1999).

[20] P. A. Lee and N. Nagaosa, Phys. Rev. B 46, 5621 (1992).

[21] D. P. Arovas and A. Auerbach, Phys. Rev. B 38, 316 (1988).

[22] J. E. Hirsch and Sanyee Tang, Phys. Rev. B 39, 2850 (1989).

[23] For small $q$, there is an additional term from condensate: $\left(\omega^{3} / \varepsilon_{f}^{2}\right)\left[n_{b}(0) / 2 S\right]\left(m_{f} / m_{b}\right)\left(k_{f} / q\right)$. It will not to affect our results because its weight in phase space is small. 